\title{
A New Prospective for Learning Automata: A Machine Learning Approach
}

\author{
Wen Jianga,, Bin Li ${ }^{\mathrm{b}}$, Shenghong Li ${ }^{\mathrm{a}, *}$, Yuanyan Tang ${ }^{\mathrm{c},}$, Chun Lung Philip \\ Chen ${ }^{\mathrm{c}}$, \\ ${ }^{a}$ Department of Electronic Engineering, Shanghai Jiao Tong University, Shanghai, 200240, \\ China \\ ${ }^{b}$ School of Information and Communication Engineering, Beijing University of Posts and \\ Telecommunications, Beijing, 100876, China \\ ${ }^{c}$ Faculty of Science and Technology, University of Macau, Macau, China
}

\begin{abstract}
In the field of Learning Automata (LA), how to design faster learning algorithms has always been a key issue. Among solutions reported in the literature, the stochastic estimator reward-inaction learning automaton $\left(S E_{R I}\right)$, which belongs to the Maximum Likelihood estimator based LAs, has been recognized as the fastest $\epsilon$-optimal LA. In this paper, we first point out the limitations of the traditional Maximum Likelihood estimator (MLE) based LAs and then introduce Bayesian estimator based approach, which is demonstrated to be equivalent to Laplace smoothing of the traditional method, to overcome these limitations. The key idea is that the Bayesian estimator, which estimates the probability of selecting each action in the LA, aims to reconstruct Bernoulli distribution from sequential data, and is formalized based on exponential conjugate family so that the LA has a relatively simple format for easy implementation. In addition, we also indicate that this Bayesian estimator could be applied to update almost all existing MLE estimator based LAs. Based on the proposed Bayesian estimator, a new LA, known as Generalized Bayesian Stochastic Estimator (GBSE) LA, is presented and proved to be $\epsilon$-optimal. Finally, extensive experimental results
\end{abstract}

\footnotetext{
* Corresponding author

Email addresses: wenjiangesjtu.edu.cn (Wen Jiang), stonebuptegmail.com (Bin Li), shli@sjtu.edu.cn (Shenghong Li), yytang@umac.mo (Yuanyan Tang), philipchen@umac.mo (Chun Lung Philip Chen)
}

Preprint submitted to Journal of ${ }^{A} T_{E} X$ Templates

April 10, 2015

C) 2015. This manuscript version is made available under the Elsevier user license http://www.elsevier.com/open-access/userlicense/1.0/ 
on benchmarks demonstrate our proposed learning scheme is more efficient than the current best LA $S E_{R I}$.

Keywords: Learning Automata, $\epsilon$-optimal, Bayesian estimator, Maximum Likelihood estimator

\section{Introduction}

Learning Automata (LA), a reinforcement learning approach as one of the most powerful tools in adaptive learning systems, is an important research area of Artificial Intelligence (AI). It has a broad range of applications as been seen

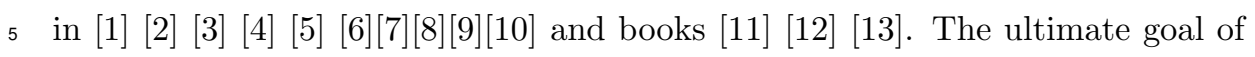
LA is to learn the sole optimal action from the environment so as to achieve higher reward compared with other actions via interacting with the environment in terms of a sequence of repetitive feedback cycles. The functionality of LA can be generally summarized as follows. Firstly, it selects an action from a predefined action set according to a probability distribution vector defined as the probability of choosing an action at each instant. Then, this selected action will trigger the environment to generate a response to the LA according to the reward probability of each action. The LA takes this response and the corresponding selected action into account to update its probability vector. This process terminates when one element of the probability vector is equal to one. By learning to choose the optimal action, the automaton adapts itself to the environment. The LA has many merits such as being applicable to stochastic environment, having solid theoretical background, owning easily deployed stop criteria and providing online learning ability. At the meantime, it does not need a predefined metric space and achieves the global optimal solutions (for certain type of LA). These advantages have been playing a vital important role to extend its applications, but the slow convergence of the LA prohibits itself from applying to a wider range of applications. Hence, one of the major goals in the LA research area is to design fast LA algorithms.

The idea of LA was first introduced by Tsetlin to model biological learning 
mechanism [14. Since then LA has been extensively studied to develop various kinds of LA algorithms. These reported LA algorithms in the literature could be classified into two categories, i.e., Continuous Action-set Learning Automata (CALA) and Finite Action-set Learning Automata(FALA). In the category of 30 FALA, one action is taken from a finite action set at each instant, while CALA takes the action from a real line (or an interval on the real line) so as to deal with continuous-valued parameters directly. So far, in the existing literatures, most of the Learning Automata (LA) we usually referred to is the FALA. Therefore, we will adopt this conventional indication in this paper.

FALA can also be further subdivided into two classes. The first one is known as Fixed Structure Stochastic Automata (FSSA) [14] [15, which has the property that its transition and output functions do not vary with time. The second class is named as Variable Structure Stochastic Automata (VSSA) whose transition and output functions change with time. So far, much effort has been made to design faster VSSA algorithms. As a pioneer researcher on LA, Oommen proposed discretized LA [16] with a convergence rate faster than their continuous counterpart. Meanwhile, Thathachar and Sastry introduced the class of Maximum Likelihood Estimator (MLE) learning automata [17 [18, [19. Simulations have been demonstrated the superiority of the Maximum Likelihood estimator based algorithm over the one without estimator. Later, Georgios et.al presented the stochastic estimator reward-inaction learning automaton $\left(S E_{R I}\right)$ based on stochastic MLE [20, which has been known to be the fastest LA so far. Another fast converging approach, known as structure LA has also been reported in [21] 22.

Generally speaking, all estimator based algorithms mentioned above are actually derived by maximum likelihood estimation. The automaton records the number of times that each action has been selected and the number of times that each action has been rewarded. Then the reward estimates will be calculated as the ratio between the number of rewarded selections and the number 55 of total selections. Recently, a new Bayesian LA (BLA) has been introduced in [23. It is also demonstrated that the corresponding Bayesian pursuit algorithm 
is superior to BLA. As mentioned in 23], both the BLA and corresponding pursuit algorithm have the beauty of relying on updating the hyper-parameters of sibling conjugate prior. Nevertheless, they still need sampling from the resulting posterior.

In this paper, we point out the limitations of MLE based LAs, and introduce Bayesian estimator to calculate the deterministic estimates of each action based on Bayesian estimation and present a Bayesian stochastic estimator scheme, which is known as the Generalized Bayesian Stochastic Estimator Learning Automata $(G B S E)$. The estimates of the reward probability of actions for the $G B S E$ are computed by stochastically varying these Bayesian estimations to relax the relationship between the stochastic and the deterministic estimates which enables the actions selected for only a few times to be optimal. Consequently, it can be found that the GBSE converges rapidly and accurately.

The key contributions of this paper are summarized as follows.

- The new and novel Bayesian estimator proposed in this paper will play a fundamental role in the field of LA. To overcome the limitations from all existing MLE based LAs and construct faster LA, we propose the Bayesian estimator and demonstrate that it is equivalent to the MLE with Laplace smoothing. We also show that this Bayesian estimator can be applied to all existing MLE based LAs in order to overcome the limitations from the MLE ones.

- We also propose a new algorithm, referred to as Generalized Bayesian Stochastic Estimator Learning Automata $(G B S E)$ and prove that the proposed scheme is $\epsilon$-optimal in all stationary environments.

- The proposed $G B S E$ is compared with by far the fastest LA, $S E_{R I}$, in various benchmark environments. Extensive experiments demonstrate that the proposed one is more efficient than $S E_{R I}$.

The rest of this paper is organized as follows. In Section 2, we shall introduce 85 the general idea of LA and the Maximum Likelihood Estimator. Section 3 
introduces the concept of the proposed Bayesian estimator. The details of the proposed GBSE LA and the $\epsilon$-optimality of this scheme are presented in Section 4. Extensive simulation results are presented to demonstrate the advantages of the proposed algorithm over the fastest known LA, $S E_{R I}$, in Section 5 . The last Section concludes this paper with some conclusive remarks.

\section{Learning Automata and ML Estimator}

Let us first introduce some notations and definitions, and then pointed out limitations of the MLE based LAs. Then the problems related to the MLE based LAs are identified.

\subsection{LA and Stochastic Environment}

The stochastic estimator LA framework is represented as a quintuple < $\mathbb{A}, \mathbb{B}, \mathbb{P}, \mathbb{E}, \mathbb{T}>$, where

- $\mathbb{A}=\left\{\alpha_{1}, \alpha_{2}, \ldots, \alpha_{r}\right\}$ is a finite set of actions.

- $\mathbb{B}=\{0,1\}$ is the response set of the P-model environment, while the Qmodel and the S-model environments are defined as $\mathbb{B}=\{0, \ldots, 1\}$ and $\mathbb{B}=[0,1]$, respectively.

- $\mathbb{P}$ is the probability distribution over actions. At instant $t$, the probability vector is defined as $\mathbf{P}(t)=\left[p_{1}(t), p_{2}(t), \ldots, p_{r}(t)\right]^{T}$, where $p_{i}(t)=p\{\alpha(t)=$ $\left.\alpha_{i}\right\}$.

- $\mathbb{E}$ is the estimator. For each action $\alpha_{i}$ at instant $t, E_{i}(t)=\left(\hat{d}_{i}(t), R_{i}(t)\right)$, in which $\hat{d}_{i}(t)$ is deterministic estimates of the reward probability of action $\alpha_{i}$ and $R_{i}(t)$ is a random variable followed with some predefined distributions.

- $\mathbb{T}$ is the learning paradigm, $\mathbb{T}:[0,1]^{r} \times \mathbb{A} \times \mathbb{B} \rightarrow[0,1]^{r}$. The probability vector is updated by the following equation:

$$
\mathbf{P}(t)=\mathbf{T}(\mathbf{P}(t), \alpha(t), \beta(t)) .
$$


The stochastic environment interacted with the LA is defined as $<\mathbb{A}, \mathbb{B}, \mathbb{D}>$, where $\mathbb{A}$ and $\mathbb{B}$ are defined above; $\mathbb{D}=\left\{d_{1}, d_{2}, \ldots, d_{r}\right\}$ is the probability distribution over actions and

$$
p\left\{\beta(t)=1 \mid \alpha(t)=\alpha_{i}\right\}=d_{i}
$$

where $\beta(t)$ is the output of the stochastic environment at each instant $t$. Throughout this paper, $\beta(t)=1$ means that the environment rewards the action selected by the LA.

From the above definition, it is easy to understand that the output $\beta(t)$ follows Bernoulli distribution given a selected action $\alpha_{i}$. It is well known that Bernoulli distribution is governed by two parameters: the number of trials and the parameter which characterizes each Bernoulli trial. On the LA counterpart, the number of trials is actually the number of times that each action is selected. So our goal is to estimate another parameter, the reward probability $d_{i}$, of Bernoulli distribution.

\subsection{Maximum Likelihood Estimator}

As mentioned above, stochastic estimator based LA makes use of the Maximum Likelihood Estimation to estimate $d_{i}$. It is known that the Maximum Likelihood Estimation produces the estimate, $\hat{d}_{i}(t)$, of the probability parameter of Bernoulli distribution as

$$
\hat{d}_{i}(t)=\frac{W_{i}(t)}{Z_{i}(t)}
$$

which asymptotically converges to the true parameter of the Bernoulli distribution, i.e.,

$$
\lim _{t \rightarrow \infty} \hat{d}_{i}(t)=d_{i}
$$

where $W_{i}(t)$ and $Z_{i}(t)$ are the total numbers of action $\alpha_{i}$ having been selected and rewarded till time instant $t$, respectively.

A key point worthy of mentioning here is that the MLE based LAs suffer from the following two problems. The first one is the cold startup problem, i.e., there is no trial and observation as the learning system starts, $W_{i}(0)=0$ 

problem underlying the traditional MLE based LA is more serious. Assuming that at time instant $t=1$, the LA selects one action $\alpha_{\text {selected }}$ ( $\alpha_{\text {selected }} \neq$ $\left.\alpha_{\text {optimal }}\right)$. Then the estimate for the optimal action will not change. However, we have $\hat{d}_{\text {selected }}(1)=1$ if the stochastic environment rewards the selected action $\alpha_{\text {selected }}$. For a subclass of the MLE based LAs, i.e., pursuit LAs which update their probability vectors to follow the maximum ML estimators, since $\hat{d}_{\text {selected }}(1)=1>\hat{d}_{\text {optimal }}(1)=0$, so the optimal action will never be selected.

The most widely used strategy to deal with these two drawbacks mentioned above is that those existing estimator based LAs MUST initialize their estimates by selecting each action for several times to the stochastic environment and recording the corresponding environment responses. However, this initialization method still has several limitations as follows.

- Under the environment whose response is expensive or very difficult to get, this solution would not be feasible.

- Considering a game of ML estimator based LAs with $N$ players or LAs, each of which has $r$ actions, the number of total combinations of actions is $N^{r}$. In order to converge to the global optimal action, a possible solution is employing the estimator algorithms 24. However, this initialization solution needs to select each combination of actions for several times in advance, which means that there are eachActionInitializingTimes $\times N^{r}$ selections at the initialization stage. This is, of course, unbearable for practical applications.

- We further point out that this method can not fundamentally solve the above mentioned two problems since the MLE of optimal action remains to be 0 with certain probability, even though this probability becomes smaller as the number of initial selections increases. Different with the deterministic estimator (MLE) based LAs, the stochastic MLE based LA can inherently overcome this problem since the probability vector is updated based on the MLE estimates plus a random variable which might 
be negative. Thus the lowest estimate value used for updating probability vector will never be 0 . However, stochastic MLE based algorithms are fundamentally distinguished from the MLE estimator based LAs and thus could not be employed to update all MLE based LAs. This paper introduces a new LA estimator based on Bayesian inference to solve the above two problems, which is also applicable to all existing MLE based LAs.

\section{Bayesian Estimator}

The Bayesian and frequentist approaches are two broad types of statistical inference [25]. Bayesian approach usually treats the unknown parameter $d_{i}$ as random variables. The frequentist approach interprets $d_{i}$ as the fixed parameters and the frequency of an event approaches this parameter as the number of tries tends to infinity. We adopt exactly the Bayesian perspective in which the Bayesian theory plays a key role as:

$$
\begin{aligned}
& p\left(d_{i}, \beta(t) \mid a(t)=a_{i}\right) \\
& =p\left(d_{i} \mid \beta(t), a(t)=a_{i}\right) \cdot\left(\beta(t) \mid a(t)=a_{i}\right)
\end{aligned}
$$

where $d_{i}$ is seen as a random variable and $\beta(t)$ is an observed value at each instant $t$. Meanwhile, the posterior distribution of $d_{i}$, given the observance of $\beta(t)$, is defined as:

$$
\begin{array}{r}
p\left(d_{i} \mid \beta(t), a(t)=a_{i}\right)=\frac{\left.p\left(d_{i}, \beta(t)\right) \mid a(t)=a_{i}\right)}{p\left(\beta(t) \mid a(t)=a_{i}\right)} \\
=\frac{p\left(d_{i} \mid a(t)=a_{i}\right) \cdot\left(\beta(t) \mid d_{i}, a(t)=a_{i}\right)}{p\left(\beta(t), a(t)=a_{i}\right)} .
\end{array}
$$

The key quantities are $p\left(d_{i} \mid a(t)=a_{i}\right)$ and $p\left(\beta(t) \mid d_{i}, a(t)=a_{i}\right)$, which are referred to as the prior distribution and likelihood function, respectively. Hence, the above equation is often written as follows

$$
\text { posterior } \propto \text { prior } \times \text { likelihood. }
$$

With the above definition, we can introduce the conjugate prior distribution defined in [25]. Let $\mathcal{H}$ be a class of sampling distribution $p(\cdot \mid \theta)$ and $\mathcal{P}$ be a class 
of prior distributions for $\theta$, then the class $\mathcal{P}$ is said to be a conjugate for $\mathcal{H}$ if

$$
p(\theta \mid \cdot) \in \mathcal{P} \text { for all } p(\cdot \mid \theta) \in \mathcal{H} \text { and } p(\cdot) \in \mathcal{P} \text {. }
$$

A special case of the above definition is the so called natural conjugate prior families. Every exponential distribution in this natural conjugate prior families, $p\left(d_{i} \mid \beta(t), a(t)=a_{i}\right)$, has a natural conjugate prior distribution. Taking the Bernoulli distribution as an example, let us consider the random variable $\beta(t) \in\{0,1\}$ so that

$$
\begin{array}{r}
p\left(\beta(t)=1 \mid d_{i}, a(t)=a_{i}\right)=d_{i} \\
p\left(\beta(t)=0 \mid d_{i}, a(t)=a_{i}\right)=1-d_{i} .
\end{array}
$$

Therefore, the Bernoulli distribution over $\beta(t)$ can be formalized as

$$
\begin{aligned}
p\left(\beta(t) \mid d_{i}, a(t)=a_{i}\right) & =d_{i}^{\beta(t)}\left(1-d_{i}\right)^{(1-\beta(t))} \\
& =\left(1-d_{i}\right) e^{\log d_{i}^{\beta(t)}\left(1-d_{i}\right)^{-\beta(t)}} \\
& =\left(1-d_{i}\right) e^{\beta(t) \log d_{i}} e^{-\beta(t) \log \left(1-d_{i}\right)} \\
& =\left(1-d_{i}\right) e^{\beta(t) \log \frac{d_{i}}{1-d_{i}}} .
\end{aligned}
$$

It is easy to see from Eq. 10 that the Bernoulli distribution is actually a member of exponential family. It is well known that the conjugate prior distribution of Bernoulli distribution is Beta distribution, i.e.,

$$
\begin{aligned}
& \operatorname{Beta}\left(d_{i} \mid \mu_{i}, \nu_{i}, a(t)=a_{i}\right) \\
& =\frac{\Gamma\left(\mu_{i}+\nu_{i}\right)}{\Gamma\left(\mu_{i}\right) \Gamma\left(\nu_{i}\right)} d_{i}^{\left(\mu_{i}-1\right)}\left(1-d_{i}\right)^{\left(\nu_{i}-1\right)} .
\end{aligned}
$$

where $\Gamma(x)$ is the gamma function. We can verify the conjugate prior of Bernoulli distribution in Eq. 10 as follows:

$$
\begin{aligned}
& p\left(d_{i} \mid \beta(t), a(t)=a_{i}\right) \\
& \propto \operatorname{Beta}\left(d_{i} \mid \mu_{i}, \nu_{i}, a(t)=a_{i}\right) p\left(\beta(t) \mid a(t)=a_{i}\right) \\
& \propto d_{i}^{\mu_{i}-1+\beta(t)}\left(1-d_{i}\right)^{\nu_{i}-1+1-\beta(t)} .
\end{aligned}
$$

As seen from Eq. [12], the posterior distribution has the same parametric 190 form as the prior distribution, i.e., the Beta distribution in Eq. 11. By comparing Eq.111 with Eq. 12, the rules of updating the parameters of the posterior 
distribution can be derived as:

$$
\begin{aligned}
& \mu_{i}(t)=\mu_{i}(t-1)+\beta(t) \\
& \nu_{i}(t)=\nu_{i}(t-1)+1-\beta(t) .
\end{aligned}
$$

Considering the fact that the expectation of Beta distribution is $\frac{\mu_{i}(t)}{\mu_{i}(t)+\nu_{i}(t)}$, we choose the estimate of $d_{i}$ as

$$
\begin{aligned}
\hat{d}_{i}(t) & =E\left\{\operatorname{Beta}\left(d_{i} \mid \mu_{i}(t), \nu_{i}(t), a(t)=a_{i}\right)\right\} \\
& =\frac{\mu_{i}(t)}{\mu_{i}(t)+\nu_{i}(t)} .
\end{aligned}
$$

Meanwhile, let us define the corresponding stochastic estimator as

$$
u_{i}(t)=\hat{d}_{i}(t)+R_{i}(t)
$$

where $R_{i}(t)$ is a random number uniformly distributed in the range of $\left(-\frac{\gamma}{\mu_{i}(t)+\nu_{i}(t)}, \frac{\gamma}{\mu_{i}(t)+\nu_{i}(t)}\right)$.

One more issue to be addressed is which type of prior distribution to be used, i.e., informative prior distribution or non-informative prior distribution. Non-information prior distribution does not have any prior belief about the parameter $d_{i}$ itself, for example, the uniform distribution and information prior hold the prior belief or knowledge on the parameter. Since the Beta distribution is reduced to a uniform distribution with $\mu_{i}(0)=1, \nu_{i}(0)=1$, the initial phase has a non-information prior distribution. Note that this initialization condition will lead to $\hat{d}_{i}(0)=\frac{1}{2}=0.5$. As no observation is available at time instant 0 205 for the case of MLE approach, $\hat{d}_{i}(0)$ does not have any meaning and cannot be calculated. This is the fundamental difference between MLE and Bayesian estimators. Therefore, the LA based on the proposed Bayesian estimator will directly initialize $\hat{d}_{i}(0)=0.5$. In this way, the above mentioned limitations of the MLE based LAs can be avoided. Because $\hat{d}_{i}(0)=\frac{1}{2}=0.5$ is the middle point in the range of $(0,1)$, it is statistically closer to the random variable $d_{i}$ than any other initial values in the range of $(0,1)$, which leads to faster convergence. 
Generally, Eq.14 can also be written as

$$
\begin{aligned}
\hat{d}_{i}(t) & =E\left\{\operatorname{Beta}\left(d_{i} \mid \mu_{i}(t), \nu_{i}(t), a(t)=a_{i}\right)\right\} \\
& =\frac{\mu_{i}(t)}{\mu_{i}(t)+\nu_{i}(t)}, \mu_{i}(0)=1, \nu_{i}(0)=1 \\
& =\frac{W_{i}(t)+1}{Z_{i}(t)+2}
\end{aligned}
$$

where $W_{i}(t)$ and $Z_{i}(t)$ are defined as the Maximum Likelihood Estimates mentioned in Eq.(3). Comparing Eq.(3) with Eq.(16), it is very interesting to see that the Bayesian estimator is actually a 'smoothed' value of the MLE. In fact, as known to all, this is called as Laplace smoothing. Furthermore, it is trivial to prove that the Bayesian estimates or MLE with Laplace smoothing asymptotically converges to the probability parameter of an event characterizing the Bernoulli distribution, i.e.,

$$
\lim _{t \rightarrow \infty} \hat{d}_{i}^{M L E}(t)=\lim _{t \rightarrow \infty} \hat{d}_{i}^{\text {Bayesian }}(t)=d_{i}
$$

\section{Generalized Bayesian Stochastic estimator Learning Automata ( $G B S E)$}

The discretized generalized pursuit algorithm (DGPA) is demonstrated to be faster than the pursuit family of Learning algorithm with remarkable performance [26. Also in [26], GPA and DGPA have been introduced to generalize the concepts of the pursuit algorithm by pursuing all the actions that have higher estimates than the current chosen one. Actually, one explaination for this phenomenon could be found in [27] where the connection between the selectionism and Bayesianism is attempted for the Linear Reward-Inaction $\left(L_{R I}\right)$ algorithm . It is concluded that in the $L_{R I}$ scheme, the action probabilities 
are redistributed towards the better-rewarded actions as the average reward increases steadily, which is very similar to the concepts underlying the GPA and DGPA. In this paper, we adopt this idea to design a new LA, i.e., Generalized Bayesian Stochastic estimator Learning Automata $(G B S E)$ and then discuss its $\epsilon$-optimality.

\subsection{The GBSE Scheme}

Our proposed $G B S E$ LA is formally described as follows.

\section{GBSEPROCEDURE}

Input : the number of actions $r$, resolution parameters $n, \gamma$ and $\Delta=\frac{1}{r n}$

Output : the optimal action

Initialize $: \mu_{i}(0)=1, Z_{i}(0)=2, i=1,2, \ldots, r$

Repeat :

Step 1:At time $t$, choose an action, $a(t)=a_{i}$, according to the probability vector $\mathbf{P}(t)=\left[p_{1}(t), p_{2}(t), \ldots, p_{r}(t)\right]^{T}$.

Step 2:Receive a feedback from stochastic environment $\beta(t) \in\{0,1\}$.

Step 3:Set $\mu_{i}(t)=\mu_{i}(t-1)+\beta(t), Z_{i}(t)=Z_{i}(t-1)+1$.

Step 4:Compute $\hat{d}_{i}(t)=\frac{\mu_{i}(t)}{Z_{i}(t)}$.

Step 5:If $\beta(t)=0$, go to Step 1 .

Step 6:Compute stochastic estimates $u_{i}(t)=\hat{d}_{i}(t)+R_{i}(t)$, where $R_{i}(t)$ is a random number uniformly distributed within $\left(-\frac{\gamma}{Z_{i}(t)}, \frac{\gamma}{Z_{i}(t)}\right)$.

Step 7:Denote $K(t)$ as the number of actions that $u_{j}(t)>u_{i}(t)$.

Step 8:Update the probability vector as follows

- For any $j, j \neq i$ such that $u_{j}(t)>u_{i}(t), p_{j}(t+1)=\min \left\{p_{j}(t)+\frac{\Delta}{K(t)}\right\}$;

- For any $j, j \neq i$ such that $u_{j}(t)<u_{i}(t), p_{j}(t+1)=\max \left\{p_{j}(t)-\right.$ $\left.\frac{\Delta}{(r-K(t))}, 0\right\}$;

260

- For current action $i, p_{i}(t+1)=1-\sum_{j \neq i} p_{j}(t)$.

Step 9: If $\max \left\{p_{j}(t+1),\{j=1 \ldots r\}\right\}=1$ then the convergence is reached, else go to Step 1. 
Table 1: accuracy (number of correct convergences / number of experiments) of $S E_{R I}$ and $G B S E$ repeated 50000 times for environments $E_{1}$ to $E_{5}$, when using the 'best' parameters

\begin{tabular}{lccccc} 
& $E_{1}$ & $E_{2}$ & $E_{3}$ & $E_{4}$ & $E_{5}$ \\
\hline$G B S E$ & 0.999 & 0.999 & 0.997 & 0.999 & 0.998 \\
$S E_{R I}$ & 0.997 & 0.997 & 0.995 & 0.998 & 0.997
\end{tabular}

\section{ENDPROCEDURE}

Definition 1. $\epsilon$-optimality: Given any arbitrarily small $\epsilon>0$ and $\delta>0$, there exist large enough $n_{0}<\infty$ (that depends on $\epsilon$ and $\delta$ ) and $t_{0}<\infty$ such that for all time $t \geq t_{0}$ and for any resolution parameter $n>n_{0}: \operatorname{Pr}\left\{\left|p_{m}(t)-1\right|<\epsilon\right\}>1-\delta$.

The flaws in the proof of $\epsilon$-optimality for pursuit learning algorithm were 
revealed in [28]. Based on 28], we shall be easily able to prove the $\epsilon$-optimality of the proposed $G B S E$. Let us first consider three important Lemmas related to the proof of the $\epsilon$-optimality.

Lemma 1. For each action $\alpha_{i}$, if $p_{i}(0) \neq 0$, then for any given constants $\delta>0$ and $M>0$, there exist $n_{0}<0$ and $T_{1}<\infty$ such that for all resolution parameters $n>n_{0}$ and all time $t>T_{1}, \operatorname{Pr}\left[\alpha_{i}\right.$ is selected more than $M$ times at time $t] \geq 1-\delta$.

For the details of the proof, please refer to [18]. This Lemma essentially means $Z_{i}(t) \rightarrow \infty$ as $t \rightarrow \infty$. Then, the MLE is to asymptomatically converge, i.e., $\hat{d}_{i}(t) \rightarrow d(t)$ as $t \rightarrow \infty$, which is formally presented in the following Lemma.

295 Lemma 2. For each action $\alpha_{i}$, if $p_{i}(0) \neq 0$, then for any arbitrarily small $\delta>0$ and arbitrarily small $\eta>0$, there exists $T_{2}<\infty$, such that, for all time $t>T_{2}$ and $i \in\{1,2, \ldots, r\}$, there have [28]:

$$
\operatorname{Pr}\left\{\left|\hat{d}_{i}(t)-d_{i}\right|>\eta\right\}<\delta
$$

Lemma 3. Suppose there exist an index $m$ and a time instant $T_{3}<\infty$ such that $u_{m}(t)>u_{j}(t)$ for all $j, j \neq m$ and all $t \geq T_{3}$, then $p_{m} \rightarrow 1$ with a unit probability as $t \rightarrow \infty$.

Proof. Lemma 3 is referred to as monotonicity property of learning algorithm and its proof is the same as Theorem 7 in [26], which is included here for convenience.

Firstly, we shall illustrate that the sequence of random variable $\left\{p_{m}(t)\right\}_{t \geq 0}$ 05 is a submartingale. The convergence will be reached from the submartingale convergence theorem [11].

Based on the aforementioned probability updating scheme in the GBSE 
algorithm, we have:

$$
\begin{aligned}
p_{m}(t+1) & =p_{m}(t)+\frac{\Delta}{K(t)}, & & \text { if } \alpha(t)=\alpha_{i}, i \neq m \\
p_{m}(t+1) & =1-\sum_{i \neq m}\left(p_{i}(t)-\frac{\Delta}{r-1}\right) & & \text { if } \alpha(t)=\alpha_{m} .
\end{aligned}
$$

Then, for $t \geq T_{3}$, we have

$$
\begin{aligned}
& E\left[p_{m}(t+1)-p_{m}(t) \mid \mathbf{Q}(\mathbf{t})\right] \\
& =\sum_{i \neq m}\left(\frac{\Delta}{K(t)}\right) \cdot p_{i}(t)+\Delta \cdot p_{m}(t) \\
& =\frac{\Delta}{K(t)}+\Delta \cdot p_{m}(t) \cdot\left(1-\frac{1}{K(t)}\right) \geq 0
\end{aligned}
$$

where $\mathbf{Q}(\mathbf{t})=(\mathbf{P}(\mathbf{t}), \mathbf{R}(\mathbf{t}), \hat{\mathbf{d}}(\mathbf{t}))$ is the state vector of our stochastic estimator algorithm.

Thus, $p_{m}(t)$ is a submartingale. From the submartingale convergence theorem[11], it can be found that the process $\left\{p_{m}(t)\right\}_{t \geq 0}$ is converged. $E\left[p_{m}(t+1)-\right.$ $\left.p_{m}(t) \mid \mathbf{Q}(\mathbf{t})\right] \rightarrow 0$ with the probability of 1 , abbreviated as w.p.1 and $p_{m}(t) \rightarrow 1$ w.p.1. Hence, Lemma 3 is proved.

With the above three Lemmas, we shall easily prove that the BGSE algorithm is $\epsilon$-optimal.

Theorem 1. In every stationary random environment, the GBSE is $\epsilon$-optimal. Formally, given any arbitrarily small $\epsilon>0$ and $\delta>0$, there exist large enough $n_{0}<\infty, t_{0}<\infty$ and a small enough $\gamma_{0}>0$ such that for all time $t \geq t_{0}$ and for any resolution parameter $n>n_{0}, \gamma<\gamma_{0}$, there have $\operatorname{Pr}\left\{\left|p_{m}(t)-1\right|<\epsilon\right\}>1-\delta$.

Proof. Let us first clarify the relationship among $u_{i}(t), \hat{d}_{i}(t)$ and $d_{i}(t)$. Set $\theta>0$ to be the difference between the largest and the second largest environment reward probabilities, $d_{m}$ and $d_{i}$. Since the reward probability of the best action is unique, it follows that $d_{m}-d_{i} \geq \theta$ for any $i \neq m, i \in\{1,2, \ldots, r\}$. 
From Lemma 2 it is known that for any action $\alpha_{i}$ and a given $\delta>0$, there

$$
\operatorname{Pr}\left\{\left|\hat{d}_{i}(t)-d_{i}\right|<\frac{\theta}{4}\right\}<\delta
$$

Denote $G_{2}^{i} \triangleq \frac{4 \gamma}{\theta}$. Considering that $R_{i}(t)$ is a random variable following uniform distribution, $U\left(-\frac{\gamma}{Z_{i}(t)}, \frac{\gamma}{Z_{i}(t)}\right)$, if $Z_{i}(t)>G_{2}^{i}$, we have:

$$
\left|R_{i}(t)\right|<\frac{\gamma}{Z_{i}(t)}<\frac{\gamma}{G_{2}^{i}}<\frac{\gamma}{4 \gamma / \theta}=\frac{\theta}{4} .
$$

Therefore, both Eq.21 and Eq. 22, will hold if $Z_{i}(t)>\max \left\{G_{1}^{i}, G_{2}^{i}\right\} \triangleq G_{3}^{i}$. In addition, it is very trivial to verify that if $Z_{i}(t)>G_{3}^{i}$, then there have [20]:

$$
\operatorname{Pr}\left\{\left|u_{i}(t)-d_{i}\right|<\frac{\theta}{2}\right\} \geq \operatorname{Pr}\left\{\left|\hat{d}_{i}(t)-d_{i}\right|<\frac{\theta}{4}\right\}>1-\delta
$$

330

which means that $u_{i}(t)$ and $\hat{d}_{i}(t)$ will be away from $d_{i}(t)$ by $\frac{\theta}{2}$ and $\frac{\theta}{4}$ with a high probability, respectively.

Next, we shall focus on proving the main Theorem. Let us define the events as follows:

$$
\begin{aligned}
A_{\epsilon}(t) & =\left\{p_{m}(t)>1-\epsilon\right\} \\
B(t) & =\left|u_{m}(t)-d_{m}\right|<\eta, t>0 \\
\bar{B}\left(T^{\prime}\right) & =\sup _{t \geq T}\left|u_{m}(t)-d_{m}\right|<\eta=\bigcap_{t \geq T} B(t) .
\end{aligned}
$$

For any $t$ and $T^{\prime}$, the following holds,

$$
\operatorname{Pr}\left\{A_{\epsilon}\left(t+T^{\prime}\right)\right\} \geq \operatorname{Pr}\left\{A_{\epsilon}\left(t+T^{\prime}\right) \mid \bar{B}\left(T^{\prime}\right)\right\} \operatorname{Pr}\left\{\bar{B}\left(T^{\prime}\right)\right\}
$$

It is known that, for $Z_{i}(t)>G_{3}^{i}, \operatorname{Pr}\left\{\left|u_{i}(t)-d_{i}\right|<\frac{\theta}{2}\right\}>1-\delta$. Then if $Z_{i}(t)>G_{3}^{i}$, there have:

$$
\operatorname{Pr}\left\{u_{m}(t)>u_{i}(t)\right\}>1-\delta, i \neq m
$$

With Lemma 3, we can derive that for those $t$ satisfying $Z_{i}(t)>G_{3}^{i}$, there have:

$$
\operatorname{Pr}\left\{A_{\epsilon}\left(t+T^{\prime}\right) \mid \bar{B}\left(T^{\prime}\right)\right\} \rightarrow 1
$$


On the other hand, $\operatorname{Pr}\left\{\bar{B}\left(T^{\prime}\right) \rightarrow 1\right.$ can be arrived by the same steps given in [28. With Lemma 2, it follows that $d_{m} \in(0,1)$ leads to $u_{m}(t) \in(0,1)$ for some $T_{4}<\infty$ and $t>T_{4}$. Then, from the inequality in Eq. (24), we have $\operatorname{Pr}\left\{A_{\epsilon}\left(t+T^{\prime}\right)\right\} \rightarrow 1$. Hence, this Theorem 1 is proved.

Table 2: the comparison of the average number of iterations required for convergence of $S E_{R I}$ and $G B S E$ in environments $E_{1}$ to $E_{5}$, when using the 'best' parameters (5000 experiments were performed for each scheme in each environment)

\begin{tabular}{ccccc}
\hline \multirow{2}{*}{ Environment } & \multicolumn{2}{c}{$G B S E$} & \multicolumn{2}{c}{$S E_{R I}$} \\
& Parameter & Iterations & Parameter & Iterations \\
\hline$E_{1}$ & $n=2, \gamma=7$ & 461 & $n=16, \gamma=8$ & 426 \\
$E_{2}$ & $n=5, \gamma=9$ & 840 & $n=32, \gamma=12$ & 834 \\
$E_{3}$ & $n=17, \gamma=17$ & 2514 & $n=105, \gamma=25$ & 2540 \\
$E_{4}$ & $n=2, \gamma=5$ & 355 & $n=13, \gamma=6$ & 325 \\
$E_{5}$ & $n=6, \gamma=8$ & 692 & $n=33, \gamma=12$ & 729 \\
\hline
\end{tabular}

Table 3: The number of iterations required to achive the same accuracy as $S E_{R I}$ shown in table 1 in environments $E_{1}$ to $E_{5}$ (5000 experiments were performed for each scheme in each environment)

\begin{tabular}{ccc}
\hline \multirow{2}{*}{ Environment } & \multicolumn{2}{c}{$G B S E$} \\
& Parameter & Iterations \\
\hline$E_{1}$ & $n=1, \gamma=7$ & 401 \\
$E_{2}$ & $n=3, \gamma=9$ & 772 \\
$E_{3}$ & $n=6, \gamma=17$ & 2262 \\
$E_{4}$ & $n=1, \gamma=5$ & 306 \\
$E_{5}$ & $n=3, \gamma=8$ & 612 \\
\hline
\end{tabular}




\section{Experiments}

In this section, we shall compare the convergence speed of the proposed $G B S E$ with that of $S E_{R I}$ which has been demonstrated to be the fastest LA in the literature 20]. As mentioned above, one of the differences between $G B S E$

$$
\begin{aligned}
E_{1}: D & =\{0.65,0.50,0.45,0.40,0.35,0.30,0.25,0.20, \\
& 0.15,0.10\} \\
E_{2}: D & =\{0.60,0.50,0.45,0.40,0.35,0.30,0.25,0.20, \\
& 0.15,0.10\} \\
E_{3}: D & =\{0.55,0.50,0.45,0.40,0.35,0.30,0.25,0.20, \\
& 0.15,0.10\} \\
E_{4}: D & =\{0.70,0.50,0.30,0.20,0.40,0.50,0.40,0.30, \\
& 0.50,0.20\} \\
E_{5}: D & =\{0.10,0.45,0.84,0.76,0.20,0.40,0.60,0.70, \\
& 0.50,0.30\} .
\end{aligned}
$$

It has been demonstrated that $S E_{R I}$ performs better than the discretized generalized pursuit algorithm (DGPA) and discretized pursuit estimator rewardinaction algorithm $\left(D P_{R I}\right)$ under the above five environments [20. In all the simulations performed, we have the same setting as [20]. For example, the computation of an algorithm is considered to converge if the probability of choosing an action is greater or equal to a threshold $T(0<T \leq 1)$. Before 
the performance comparison of different methods, many evaluation tests were executed to determine the 'best' learning parameters for each scheme. The value is considered as 'best' if it yields the fastest convergence and the automaton always converges to the correct action in a sequence of $N E$ experiments. The values of $T$ and $N E$ are taken to be equal to the ones used in [20], that is, $T=0.999$ and $N E=750$. The variance coefficient, defined as $\frac{\sigma\left\{p_{1}(t)\right\}}{E\left\{p_{1}(t)\right\}}$, where $\sigma\left\{p_{1}(t)\right\}$ is the standard deviation and $E\left\{p_{1}(t)\right\}$ is the mean of random variable $p_{1}(t)$, should be small enough since a high variance coefficient results in an unfair comparison.

On one hand, the average numbers of iterations for both schemes of the $G B S E$ and the $S E_{R I}$ are summarized in Table ??. As mentioned above, the proposed scheme do not need sample each action while $S E_{R I}$ needs sample process and these sample times are included in the table, which shows that our proposed $G B S E$ algorithm converges with the same speed as the $S E_{R I}$.

On the other hand, Table 1 demonstrates that the accuracy of the proposed $G B S E$ is higher than that of $S E_{R I}$ in all benchmark environments. For example, in Environment $E_{3}, G B S E$ takes 2514 iterations to converge while $S E_{R I}$ takes 2538 iterations; However, the accuracy of $G B S E$ is 0.997 while that of $S E_{R I}$ is 0.995. Similarly, $G B S E$ converges in 840 iterations while $S E_{R I}$ converges in 831 iterations, and the accuracy of $G B S E$ is 0.999 compared to that of $S E_{R I}$ is 0.997 in environment $E_{4}$.

380

In summary, $G B S E$ is more efficient than $S E_{R I}$. For all the environments, $G B S E$ converges with a comparable number of iterations with $S E_{R I}$ while achieving a higher accuracy, which leads to unfair comparison regarding the number of iterations.

To verify the number of iterations required with the same accuracy, a set of 385 experiments have been conducted. The results are demonstrated in Table ??, which shows that the average numbers of iterations of $G B S E$ is less than $S E_{R I}$ with the same accuracy.

Hence, the advantage of the proposed algorithm is obvious. 


\section{Conclusions}

390

The Bayesian estimator was proposed to overcome these limitations for a faster

is the Laplace smoothing version of the Maximum Likelihood estimates. A new LA algorithm based on Bayesian estimator, referred as $G B S E$, was presented and proved to be $\epsilon$-optimal in all stationary environments. Experiments have confirmed that the Bayesian estimator plays an important role in LA and shown that our proposed $G B S E$ is more efficient than the $S E_{R I}$, which is known to be the fastest LA reported in the past literatures. Since this Bayesian estimator is also the Laplace smoothing Maximum Likelihood estimates, we are currently studying how to apply this method to all other existing MLE based LAs.

\section{Acknowledgement}

This research work is funded by the National Science Foundation of China (61271316), Key Laboratory for Shanghai Integrated Information Security Management Technology Research, and Chinese National Engineering Laboratory

for Information Content Analysis Technology.

\section{References}

[1] D. S. Huang, W. Jiang, A general cpl-ads methodology for fixing dynamic paramters in dual environments, IEEE Transactions on Systems, Man, and Cybernetics-Part:B Cybernetics 42 (5) (2012) 1489-1500.

[2] W. Jiang, D. S. Huang, General framework for parameter learning and optimization in stochastic environments, IEEE Transactions on Neural Networks and Learning Systems, under review.

[3] B. Oommen, M. Hashem, Modeling a student-classroom interaction in a tutorial-like system using learning automata, IEEE Transactions on Sys- 
tems, Man, and Cybernetics-Part:B Cybernetics 40 (1) (2010) 29-42. doi:10.1109/TSMCB.2009.2032414.

[4] H. Beigy, M. Meybodi, Cellular learning automata with multiple learning automata in each cell and its applications, IEEE Transactions on Systems, Man, and Cybernetics-Part:B Cybernetics 40 (1) (2010) 54 -65. doi: $10.1109 /$ TSMCB.2009.2030786.

[ [5] M. Esnaashari, M. R. Meybodi, Data aggregation in sensor networks using

口 learning automata, Wirel. Netw. 16 (3) (2010) 687-699. doi:10.1007/ s11276-009-0162-5.

URL http://dx.doi.org/10.1007/s11276-009-0162-5

425 [6] J. G. Vlachogiannis, Probabilistic constrained load flow considering integration of wind power generation and electric vehicles, Power Systems, IEEE Transactions on 24 (4) (2009) 1808-1817.

[7] Y. Song, X. Zhu, Y. Fang, H. Zhang, Threshold optimization for rate adaptation algorithms in ieee 802.11 wlans, Wireless Communications, IEEE Transactions on 9 (1) (2010) 318-327.

[8] N. Baba, Y. Mogami, A relative reward-strength algorithm for the hierarchical structure learning automata operating in the general nonstationary multiteacher environment, Systems, Man, and Cybernetics, Part B: Cybernetics, IEEE Transactions on 36 (4) (2006) 781-794.

[9] C. Liaskos, A. Xeros, G. I. Papadimitriou, M. Lestas, A. Pitsillides, Balancing wireless data broadcasting and information hovering for efficient information dissemination, Broadcasting, IEEE Transactions on 58 (1) (2012) $66-76$.

[10] J. G. Vlachogiannis, Simplified reactive power management strategy for complex power grids under stochastic operation and incomplete information, Energy Conversion and Management 50 (12) (2009) 3193-3201. 
[11] K. S. Narendra, M. A. L. Thathachar, Learning Automata: An Introduction, Prentice-Hall, Inc., Upper Saddle River, NJ, USA, 1989.

[12] K. Najim, A. S. Poznyak, Learning Automata: Theory and Applications, Pergamon Press, Inc., Elmsford, NY, USA, 1994.

[13] A. S. Poznyak, K. Najim, Learning Automata and Stochastic Optimization, Berlin, Germany: Springer-Verlag, 1997.

[14] M. L. Tsetlin, Automaton Theory and the Modeling of Biological Systems, New York: Academic, 1973.

[15] Anastasios, A. Economides, A. Kehagias, The star automaton: Expediency and optimality properties, IEEE Transactions on Systems, Man, and Cybernetics-Part:B Cybernetics 32 (6) (2002) 723-737.

[16] B. J. Oommen, Absorbing and ergodic discretized two-action learning automata, IEEE Transactions on Systems, Man, and Cybernetics-Part:B Cybernetics SMC-16 (2) (1986) 282-293.

[17] M. A. L. Thathachar, P. S. Sastry, A new approach to the design of reinforcement schemes for learning automata, IEEE Transactions on Systems, Man, and Cybernetics SMC-15 (5) (1985) 168-175.

[18] J. K. LanctBt, B. J. Oommen, Discretized estimator learning automata, IEEE Transactions on Systems, Man, and Cybernetics 22 (6) (1992) 14731483.

[19] B. J. Oommen, M.Agache, Continuous and discretized pursuit learning schemes: Various algorithms and their comparison, IEEE Transactions on Systems, Man, and Cybernetics 31 (3) (2001) 277-287.

465 [20] I. P. Georgios, M. S. andS. Pomportsis Andreas, A new class of $\epsilon$-optimal learning automata, IEEE Transactions on Systems, Man, and CyberneticsPart:B Cybernetics 34 (1) (2004) 246-254. 
[21] A. Ansari, George, P. Papavassilopoulos, A generalized learning algorithm for an automaton operating in a multiteacher environment, IEEE Transactions on Systems, Man, and Cybernetics-Part:B Cybernetics 29 (5) (1999) 592-600.

[22] I. P. Georgios, Hierarchical discretized pursuit nonlinear learning automata with rapid convergence and high accuracy, IEEE Transactions on Knowledge and Data Engineering 6 (4) (1994) 654-659.

[23] W. Yuan, H. Leung, W. Cheng, S. Chen, Optimizing voting rule for cooperative spectrum sensing through learning automata, IEEE Transactions on Vehicular Technology 60 (7) (2011) 3253-3264.

[24] M. A. Thathachar, P. S. Sastry, Networks of learning automata: Techniques for online stochastic optimization, Springer, 2004.

[25] T. Brdland, , T. Norheim, Empirical evaluation of the bayesian learning automaton family, Master's thesis, univerisity AGDER (2009).

[26] A. Mariana, B. J. Oommen, Generalized pursuit learning schemes:new families of continuous and discretized learning automata, IEEE Transactions on Systems, Man, and Cybernetics-Part:B Cybernetics 32 (6) (2002) 738-749.

[27] J. Zhang, Adaptive learning via selectionism and bayesianism, part i: Connection between the two, Neural Networks 22 (3) (2009) 220-228.

[28] R. Martin, O. Tilak, On $\varepsilon$-optimality of the pursuit learning algorithm, Journal of Applied Probability 49 (3) (2012) 795-805. 